\section{KENTRON}

REVUE PLURIDISCIPLINAIR

DU MONDE ANTIQUE

\section{Kentron}

Revue pluridisciplinaire du monde antique

$31 \mid 2015$

Les Socratica de Xénophon

\title{
Remarques sur la megalègoria de Socrate et le tumulte des juges
}

\section{Pierre Pontier}

\section{(2) OpenEdition}

\section{Journals}

Édition électronique

URL : http://journals.openedition.org/kentron/305

DOI : $10.4000 /$ kentron.305

ISSN : 2264-1459

Éditeur

Presses universitaires de Caen

\section{Édition imprimée}

Date de publication : 1 novembre 2015

Pagination : $59-74$

ISBN : 978-2-84133-747-7

ISSN : 0765-0590

\section{Référence électronique}

Pierre Pontier, «Remarques sur la megalègoria de Socrate et le tumulte des juges », Kentron [En ligne], 31 | 2015, mis en ligne le 19 octobre 2016, consulté le 17 novembre 2020. URL : http:// journals.openedition.org/kentron/305; DOI : https://doi.org/10.4000/kentron.305

\section{(c) (i) (9)}

Kentron is licensed under a Creative Commons Attribution-NonCommercial-NoDerivatives 3.0 International License. 


\section{REMARQUES SUR LA MEGALĖGORIA DE SOCRATE ET LE TUMULTE DES JUGES}

Il me semble qu'il vaut la peine de rappeler comment Socrate, après avoir été cité

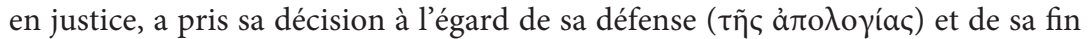
de vie. Il est vrai que d'autres ont déjà écrit sur ce sujet, et tous ont reproduit son

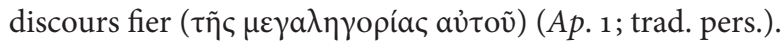

Comme on le voit dans ces lignes, l'Apologie de Socrate n'a pas pour but de rapporter ou de récrire l'intégralité des propos que Socrate aurait tenus lors de sa défense face aux juges. Le choix ${ }^{1}$ de propos mis en scène cherche à leur donner un sens par rapport à ce qu'en ont rapporté d'autres auteurs, de façon semble-t-il homogène, et ce sont ces propos que Xénophon qualifie de megalègoria ${ }^{2}$. Le plan de l'œuvre est simple. Xénophon, absent au moment du procès de Socrate, s'appuie sur le témoignage d'Hermogène pour rapporter d'abord une conversation entre le maître et son disciple: Socrate justifie son choix de ne pas préparer sa défense par une double opposition de son daimonion $(\$ 2-9)$. On trouve ensuite une sélection de ses propos lors de son procès, lesquels répondent successivement aux deux chefs d'accusation d'impiété et de corruption de la jeunesse ( $\$ 10-26)$, et, en diptyque, une évocation de l'attitude de Socrate après le procès par rapport à celle de son accusateur Anytos $(\$ 27-31)$, avant une courte récapitulation et une conclusion $(\$ 32-34)$. C’est sur le terme de megalègoria, qui définit la nature de l'apologia socratique et qui décide de l'orientation de l'opuscule, que nous aimerions faire quelques remarques.

L'étymologie de ce composé est claire: la deuxième partie du mot renvoie à une prise de parole plutôt publique ${ }^{3}$; la première partie du terme rapproche le substantif

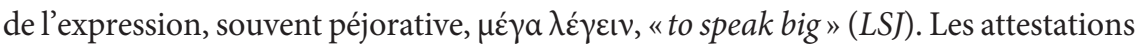
du sens rhétorique du terme («emphase») sont postérieures à l'époque classique 4 .

1. Cf. $\$ 22$ : «Mais je ne me suis pas soucié de rapporter tout ce qui s’est dit dans ce procès».

2. Voir Gray 1989 sur l'arrière-plan rhétorique possible de cette idée.

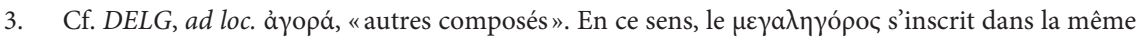

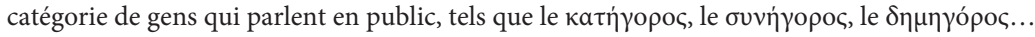

4. Voir, par ex., Demetrius, De Eloc. 29; Ps.-Long. 8, 4; 15, 1; 16, 1.

Kentron, $\mathrm{n}^{\circ} 31-2015$, p. 59-74 
La diversité des traductions proposées est la preuve des difficultés d'interprétation rencontrées par les traducteurs et les commentateurs.

Les traducteurs français de l'œuvre depuis le XVIII ${ }^{e}$ siècle ont généralement choisi de dédoubler la traduction. E. Talbot propose ainsi «la noble fierté de son langage», traduction que P. Chambry et F. Ollier reprennent de façon plus ou moins abrégée ${ }^{5}$. V. Gray propose à la fois «highmindedness» et «big talk» ${ }^{6}$; d'autres prennent plus nettement position, comme O.J. Todd, qui choisit «loftiness», et, d'un autre côté, L. Shero et P. Vander Waerdt, qui préfèrent "boastfulness ${ }^{7}$, solution que L.-A. Dorion transpose en français par le terme "vantardise ${ }^{8}$. B. Hogenmüller choisit «arrogante Großsprecherei ${ }^{9}$, tout comme G. Danzig («arrogant speech») ${ }^{10}$. Les difficultés des traducteurs témoignent de trois orientations: l'une privilégie la posture de Socrate et son ton (sa «hauteur de vues»); l'autre s'attarde plus sur l'éloge de ses actes ( «vantardise»); la troisième préfère considérer la façon dont le discours est reçu et perçu par l'auditeur (son «arrogance»). La traduction révèle donc un choix herméneutique ${ }^{11}$.

L.-A. Dorion, qui a récemment consacré un article important à la megalègoria ${ }^{12}$, interprète ainsi le terme:

Comme la megalègoria ne désigne pas tant le ton adopté par celui qui parle, que le fait même de s'attribuer ouvertement de grands mérites, c'est le terme "vantardise » qui paraît le plus adéquat pour traduire megalègoria. Dans la mesure où la megalègoria consiste à se louer soi-même pour les vertus et les mérites que l'on croit posséder, et où Socrate, par ailleurs, ne veut ni ne peut se défendre autrement qu'en insistant sur l'exemplarité de sa vie, la megalègoria qu'il affiche à son procès n'est pas autre chose qu'une forme de vantardise qui a pour but d'exalter l'ergon de sa vie ${ }^{13}$.

Il insiste à juste titre sur le rôle du daimonion, lequel permet à Socrate de «réconcilier le logos "non rhétorique" de sa défense et l'ergon de sa vie».

Nous sommes d'accord avec cette analyse. La megalègoria consiste bien, dans le cas de Socrate, à faire, en guise d'apologie, l'éloge de sa vie. Le terme de "vantardise»

5. Voir Talbot 1859, Chambry 1967, Ollier 1961 ( «fierté de son langage», $\$ 1$, mais quelques lignes plus haut, il traduit par la «hauteur de son langage»).

6. Voir Gray 1989 et LSJ, ad loc., «big talking».

7. Voir Todd 1922, 489; Shero 1927, 108; Vander Waerdt 1993, 14.

8. Voir Dorion 2013, 306.

9. Voir Hogenmüller 2008, 220-225, et Hogenmüller 2011, 176 (et note 32).

10. Danzig 2010, 25.

11. Pucci 2002, 22-25, estime qu'il faut envisager deux connotations pour ce terme, "a negative, unflattering one, and a positive, flattering one».

12. Il s'agit de l'étude la plus complète et la plus fine sur la question.

13. Voir Dorion 2013, 306. 
a certes ses limites: surtout péjoratif en français (son étymologie latine renvoie à

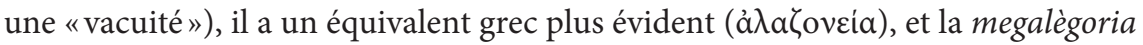
est dans le contexte de l'Apologie une forme de discours public, en l'occurrence l'apologia mentionnée dans les tout premiers mots. Mais, comme on l'a vu, toute autre solution, y compris celle que nous avons adoptée précisément pour cette raison («discours fier»), implique un dédoublement de traduction.

Quoi qu'il en soit, il est vrai que la megalègoria de Socrate, provoquée au bon moment par le daimonion, est légitimée d'un point de vue religieux ${ }^{14}$. Nous aimerions aborder la megalègoria sous un angle différent, complémentaire, à la fois éthique et rhétorique, en reprenant l'histoire du terme à l'époque de Xénophon et en analysant la façon dont l'auditoire de Socrate perçoit son discours. En effet, Xénophon comme Platon mettent en valeur les réactions des juges à plusieurs reprises. Les points de convergence comme les menues différences permettent de mieux comprendre les enjeux de la megalègoria socratique.

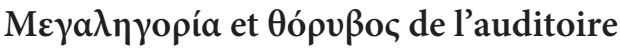

Si l'on cherche à comprendre le sens du terme à l'époque classique, on n'a d'autre choix que de se fonder sur deux auteurs tragiques, Eschyle et Euripide. La première attestation de l'adjectif $\mu \varepsilon \gamma \alpha \lambda \eta \gamma \gamma$ ó un chœur de femmes thébaines, commentant les propos tenus par les guerriers agresseurs, exprime sa peur et maudit les vantards. Le chœur vise plus précisément le cinquième, Parthénopée l'Arcadien, dont le messager vient de rapporter qu'il jure par sa lance d'abattre la cité. La jactance est ici associée à l'impiété, le guerrier lançant un défi à Zeus et aux dieux. Un peu plus loin, le messager dira que les «vantardises»

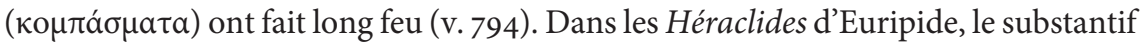
est attesté, au pluriel, dans les paroles du chœur des vieillards de Marathon. Alors que Iolaos est venu en Attique solliciter la protection pour lui et pour les enfants d'Héraclès, le héraut d'Eurysthée tente d'arracher le vieillard et les enfants à l'autel de Zeus et menace de revenir anéantir Athènes avec les armées argiennes. Après sa sortie de scène, le chœur commente les propos qu'il vient de tenir, les jugeant comme des «vantardises» (v. 356) qui expriment la bravade d'un guerrier soucieux d'établir un rapport de force.

Les deux textes présentent plusieurs points communs: les personnages - étrangers et extérieurs à la cité - qui font preuve de megalègoria cherchent à faire peur par

14. Selon Dorion 2013, 313-316, cette légitimation religieuse de la megalègoria par le daimonion avant le procès constitue une spécificité de l'Apologie de Xénophon. Son absence dans l'Apologie platonicienne pourrait être à l'origine du désir de Xénophon d'y apporter quelques correctifs. Voir aussi Dorion 2011b, 220, n. 7, et Narcy 2005, 117-119, sur le lien entre la défense de Socrate et le signe divin, ainsi que Dorion 2013, 275-300, sur ce que représente le daimonion pour Xénophon. 
leurs propos qui anticipent - à tort - leur victoire. Par ailleurs, les commentateurs considèrent leur langage comme impie ou manquant de piété, voire déraisonnable. Enfin, le constat de megalègoria est fait dans les deux cas par un chœur qui commente des propos. Les deux exemples marquent une appréciation négative des destinataires, qui n'est pas sans rappeler la connotation péjorative de l'expression $\mu \varepsilon ́ \gamma \alpha \lambda \varepsilon^{\prime} \gamma \varepsilon \nu^{15}$.

Qu'est-ce qui rapproche la megalègoria des personnages d'Euripide et d'Eschyle de celle de Socrate? Il y a d'abord la confrontation verbale d'un individu et d'un groupe. De plus la megalègoria socratique est un constat de vantardise fait par les commentateurs ou auditeurs, qu'ils aient écrit sur le procès ou fait partie des juges ou du public du procès. La dimension religieuse y est essentielle aussi, comme nous le verrons.

Dans le corpus de Xénophon, la megalègoria, présente essentiellement dans un contexte militaire, est plus ambivalente. Dans l'Anabase, Xénophon raconte d'abord, sans commentaire narratif, la sécession des Arcadiens et des Achéens près d'Héraclée; quelque temps plus tard, une fois que ces derniers sont assiégés par les Thraces et que lui-même avec sa propre troupe vient à leur rescousse, il prononce un discours destiné à ses soldats, interprétant les propos tenus par les sécessionnistes comme autant de vantardises inconséquentes dont les auteurs ont été punis après coup par les dieux:

Marchons donc, bien convaincus en notre for intérieur qu'il s'agit cette fois ou de mourir glorieusement, ou d'accomplir un bel exploit, en sauvant un si grand nombre de Grecs. Et peut-être que c'est la divinité qui mène ainsi les choses: les

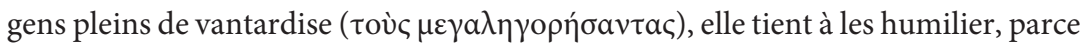
qu'ils ont une trop haute idée d'eux-mêmes; nous, au contraire, qui commençons par consulter les dieux (ảंò $\tau \tilde{\omega} v \theta \varepsilon \tilde{\omega} v$ ), ils veulent nous conférer plus d'honneurs qu'à nos camarades (trad. Masqueray 1931, 101, modifiée) ${ }^{16}$.

Le participe vise directement les Arcadiens et les Achéens (avec leurs chefs, Callimaque de Parrhasie et Lycon d'Achaie), et en particulier leurs propos tels que Xénophon les rapporte quelques pages plus haut (VI, 2, 10-11), lorsqu'ils affirmaient entre eux qu'il était honteux d'être dirigé par un Athénien alors que c'étaient eux qui, par leur nombre, avaient assuré le salut de l'armée. L'excès de confiance en soi s'accompagne apparemment dans ce cas de négligences à l'égard des devoirs de piété qui précèdent nécessairement toute prise de décision. L'action divine qui vise à rétablir un équilibre entre l'excès de fierté et l'humilité de la piété est en accord avec

15. Hom., Od. 3, 227; 16, 243; 22, 288.

16. Cf. Anabase, VI, 3, 17-18. Le cas de la Cyropédie, IV, 4, 2, est plus ambigu: les Mèdes se vantent auprès de Cyrus de leurs actes de guerre et du butin amassé, et Cyrus les félicite. Mais il est clair que les Mèdes ne sont pas des modèles de conduite morale dans la Cyropédie. 
la vision de l'histoire que Xénophon développe souvent, notamment son relativisme concernant ce qui est "grand» et ce qui est " petit» ${ }^{17}$.

En revanche, les circonstances peuvent contraindre les héros de Xénophon à faire preuve de megalègoria à bon escient. Ainsi, Agésilas, qui est décrit comme l'être le moins enclin du monde à se vanter ( $\mu \varepsilon \gamma \alpha \lambda \eta \gamma o \rho \varepsilon \tilde{v} v)$, écoute sans colère ceux qui font l'éloge d'eux-mêmes, car ce sont à ses yeux des hommes valeureux en puissance (Ages. VIII, 2) ${ }^{18}$. Lui-même, selon Xénophon, a fait preuve de «hauteur de

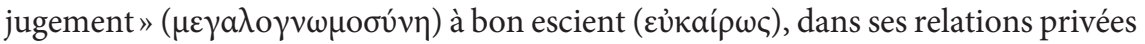
avec le Grand Roi (Ages. VIII, 3). L'exemple de Cyrus dans la Cyropédie est aussi un cas de force majeure. Avant la bataille décisive près de Babylone, lorsqu'il donne des conseils à Abradatas, Cyrus s'appuie sur le soutien des dieux pour anticiper par ses propos la fuite des ennemis:

«Si tout va bien de ton côté, rassure-toi pour nos flancs; car, avec l'aide des dieux

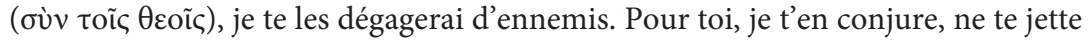
pas sur l'ennemi avant d'avoir vu fuir ceux que tu crains à présent. » Cyrus parlait

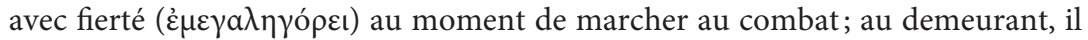
n'était pas du tout vantard ( $\mu \varepsilon \gamma a \lambda \eta \gamma$ ó $о \varsigma)$ (Cyr. VII, 1, 17; trad. Chambry 1967, 236, modifiée).

Xénophon commente cette prise de parole: la raison pour laquelle Cyrus, peu coutumier de cette attitude, se montre megalègoros, c'est que la bataille décisive est sur le point de commencer, ce qui est en soi une bonne occasion (VII, 1, 17) ${ }^{19}$. Cyrus se distingue aussi des Arcadiens de l'Anabase, puisqu'il sollicite un appui divin là où les Arcadiens, au dire de Xénophon, ne s'en préoccupent pas, ne comptant que sur eux-mêmes. En résumé, s'il n'est pas bon de toujours s'exprimer ainsi, le kairos et la relation privilégiée que l'on entretient avec les dieux peuvent légitimement imposer la megalègoria comme la meilleure des attitudes à adopter à un moment donné.

17. On pense par exemple à son éloge de la petite cité de Phlionte dans les Helléniques (VII, 2); voir aussi, d'un point de vue stylistique, Gray 2014.

18. Les passages de l'Agésilas VIII, 2 et de la Cyropédie IV, 4, 2-3 sont parallèles: la réaction des deux chefs est identique.

19. Comme dans le cas d'Agésilas, Xénophon précise que Cyrus n'était pas vraiment de nature à se vanter de façon générale (VIII, 1, 17). Seule, l'occasion - la nécessité de galvaniser ses troupes - le pousse à parler ainsi. Voir également Demont 2014, 200. Plutarque, Mor. 545b, cite aussi ce passage de la Cyropédie et semble même gloser le passage cité de l'Anabase dans les lignes qui précèdent: «[...] quant à nos amis et à nos concitoyens, il nous est possible non seulement de rabaisser ceux qui font les fiers et de les rendre plus humbles, mais aussi, inversement, d'exalter ceux qui vivent dans la crainte et dans l'effroi, et de les stimuler, en usant à propos d'un ton plein d'orgueil. C'est ainsi que, dans les dangers et les batailles, Cyrus "parlait avec fierté", mais ailleurs, il n'était "pas fier du tout" " (traduction Klaerr 1974, 78-79). 
Xénophon réserve cependant l'emploi du substantif ${ }^{20}$ à l'Apologie. Nous pensons que cela s'explique par le contexte public du discours de Socrate. Peut-être d'autres auteurs l'avaient-ils utilisé avant lui pour qualifier les propos socratiques, ou peut-être l'a-t-il déduit de la version platonicienne de l'Apologie ou d'autres témoignages. En tout cas, le substantif megalègoria s'impose, nous l'avons vu dans l'incipit, en tant que forme spécifique d'apologia, différenciant ainsi la performance de Socrate des autres cas du corpus. Il s'agit d'une forme particulière de défense, qui est propre à indisposer les juges, ce que Socrate pressent avant même le procès $(\$ 9)$.

Or, dans l'Apologie platonicienne, Socrate signale aussi dès le début le caractère particulier de sa défense, qui se différencie de la rhétorique usuelle du genre apologétique: il est bien conscient des réactions négatives qu'elle pourrait susciter au point de les prévenir à plusieurs reprises (17d, 20e-21a, 34c-d, 36d-37a).

Le deuxième de ces passages proleptiques (20e-21a) est celui qui entre le plus en convergence avec l'Apologie de Xénophon, comme on l'a déjà noté ${ }^{21}$. Platon y mentionne la réaction - réelle ou supposée - des juges sous la forme d'une double requête qui les invite à ne pas faire du bruit:

N'allez pas faire du bruit $(\theta o \rho u \beta \eta ́ \sigma \eta \tau \varepsilon)$, Athéniens, même si je vous parais tenir

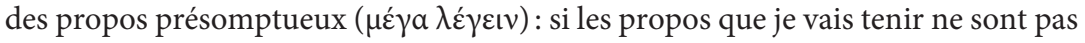
de moi, ce sont les propos de quelqu'un que vous estimez digne de foi que je vais évoquer. En effet, pour ce qui est de mon savoir - de son existence et de sa nature - , je produirai devant vous comme témoin le dieu de Delphes. Vous connaissez sûrement Chéréphon, je suppose. Ce fut pour moi un ami d'enfance et pour vous un ami du peuple: il prit part à l'exil que vous avez connu et il en revint avec vous. Vous savez bien aussi l'homme qu'il était, et le degré de passion qu'il mettait dans tout ce qu'il entreprenait. En particulier, un jour qu'il s'était rendu à Delphes, il osa consulter l'oracle sur le point suivant - et n'allez pas, citoyens, couvrir de bruit

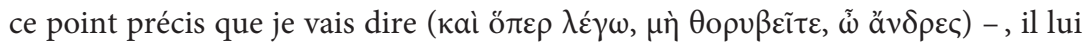
demanda donc s'il y avait quelqu'un de plus savant que moi. Or, la Pythie répondit que nul n'était plus savant. Et sur ces propos, c'est son frère que voici qui portera témoignage devant vous, puisque Chéréphon est mort (trad. Brisson 1997 modifiée).

L'expression mega legein ${ }^{22}$ utilisée au début du passage fait songer au constat unanime de megalègoria relevé par Xénophon dans les premières lignes de l'Apologie.

20. Voir Richards 1898, 193: "The very word is distinctly xenophonean ", et le bilan de Dorion 2013, 305, qui ne fait pas de distinction entre les emplois de l'adjectif, du verbe ou du nom.

21. Voir par exemple Arnim 1923, 15 et Waterfield 2012, 274.

22. L'expression est assez peu présente dans le corpus platonicien: Phédon, 95b, 101c; Théètète 152d; Banquet, 198d, 212d; Phèdre, 26od; Alcibiade 110b; République 449b. Voir aussi Waterfield 2012, 274. 
Or, l'avertissement préventif survient au moment où Socrate annonce qu'il va faire comparaître un "témoin digne de foi», le dieu de Delphes. Dans les deux autres passages platoniciens ( $34 \mathrm{c}-\mathrm{d}, 36 \mathrm{~d}-37 \mathrm{a})$, il indique qu'il n'aura pas recours aux supplications ou qu'il mérite d'être nourri au prytanée, et il essaie de se défendre contre l'impression d'arrogance que son discours peut laisser, sans toutefois inviter les juges à ne pas faire de tumulte.

Dans la version de Xénophon, le tumulte et les exclamations des juges sont particulièrement mis en valeur par l'effet dramatique de la double interruption des propos de Socrate commentée par le narrateur, cas unique dans l'Apologie:

«Et voici la preuve que je ne mens pas en faisant injure à la divinité: j’ai révélé à nombre de mes amis les avertissements que j'avais reçus d'elle sans avoir jamais été convaincu de mensonge à cet égard.»

Comme en entendant ces mots les juges faisaient du tumulte ( $\dot{\varepsilon} \theta$ opúßouv), les uns par défiance à l'égard des propos de Socrate, les autres par jalousie surtout de ce qu'il obtînt même de la part des dieux plus qu'eux-mêmes, Socrate reprit la parole:

"Allons, écoutez encore autre chose, pour que ceux d'entre vous qui le désirent se défient encore davantage de la faveur dont m'ont honoré les dieux. Un jour que Chéréphon, à Delphes, interrogeait l'oracle à mon sujet en présence de nombreux témoins, Apollon répondit que personne n'était plus désintéressé que moi, ni plus juste, ni plus sage.»

Comme les juges, naturellement, faisaient plus de tapage encore ( $\dot{\varepsilon} \theta$ o $u ́(\beta o u v)$ à ces mots, Socrate continua de la sorte: «Pourtant, juges, le dieu par la voix de l'oracle s'est exprimé plus magnifiquement sur le compte de Lycurgue,

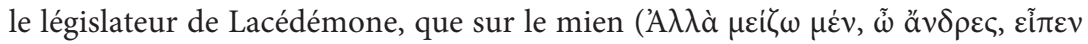

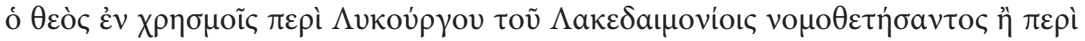
$\dot{\varepsilon} \mu o \tilde{v})$. On raconte en effet qu'il lui dit, quand Lycurgue entrait dans son temple: «Je me demande si je dois t'appeler un dieu ou un homme». Pour moi, sans me comparer à un dieu, il a pourtant jugé que je l'emportais de beaucoup sur le reste des hommes.» (Ap. 13-15; trad. Ollier 1961, modifiée).

Cette double notation narrative du tumulte des juges, dans un contexte proche de l'Apologie platonicienne, invite à rapprocher les deux passages: il s'agit d'un même effet dramatique, souligné par deux procédés d'écriture différents.

La megalègoria de Socrate a pour effet de susciter la réaction bruyante de son auditoire, à ce moment précis de l'Apologie de Xénophon. L'auteur y fait discrètement allusion dans un jeu de mots destiné à introduire l'exemple de Lycurgue: le comparatif $\mu \varepsilon i \zeta \omega$ placé en début de réplique, dans l'expression $\mu \varepsilon i ́ \zeta \omega \lambda \varepsilon^{\prime} \gamma \varepsilon \imath v$, permet de qualifier la façon dont le dieu se serait exprimé sur le compte du législateur lacédémonien. La megalègoria suprême est donc placée dans la bouche d'Apollon, comme dans le texte platonicien. 
De cette première approche croisée de la megalègoria socratique, on retiendra trois points. Son emploi est isolé à l'époque classique, Xénophon étant le seul à utiliser le substantif au singulier. De plus, le «tumulte» (thorubos) constitue un signe tangible de réaction du public à la megalègoria. Comme dans les exemples tragiques, elle semble impliquer un rapport de force ou de défiance entre un orateur et un groupe, son auditoire. Les deux Apologies dramatisent la scène, en montrant le lien sémantique entre mega legein ou megalègoria et le thorubos. Le sommet dramatique correspond dans les deux cas à la révélation de la parole de l'oracle, même si la megalègoria socratique ne se limite pas à ces quelques lignes.

\section{Megalègoria, éloge de soi et jalousie}

Mais l'approche de Xénophon présente trois spécificités:

1. À la différence du Socrate platonicien, qui préfère à ce moment-là rappeler qui était Chéréphon et son orientation politique démocratique, le Socrate de Xénophon invoque le cas de Lycurgue, se comparant donc par oracle interposé au fondateur mythique de Sparte. Était-ce perçu par le public athénien comme une provocation délibérée après trente ans de guerre contre Sparte ${ }^{23}$ ? Cela est fort possible, même si l'histoire était bien connue ${ }^{24}$. En tout cas, Socrate ne s'assimile ni à Lycurgue ni à un dieu, tout en soulignant qu'il occupe, selon le dieu, un rang intermédiaire entre les hommes et les dieux. La comparaison sert surtout le propos de Xénophon, qui cherche à montrer que Socrate, par son régime de vie, essaie de se rapprocher le plus possible de la conduite d'un dieu ${ }^{25}$. Il propose une autre interprétation de la megalègoria socratique que celle que l'on voit à l'œuvre dans l'A pologie platonicienne.

2. Les propos qui déclenchent initialement le tumulte sont d'une teneur très différente: Socrate parle de la divinité dans la version de Xénophon, alors que Platon met en avant l'affirmation que Socrate possède une anthrôpinè sophia, une «science humaine» ou une «science propre à l'homme» ${ }^{26}$. Chez Xénophon, Socrate insiste sur la relation spécifique qu'il entretient avec le dieu, laquelle lui permet d'avertir ses amis de l'avenir et de leur donner des conseils infaillibles: tels sont les propos qui suscitent à la fois l'incrédulité et la jalousie (phthonos) des juges. Chez Platon, en revanche, Socrate essaie de prévenir ou d'anticiper par ses propos le tumulte à

23. C'est l'opinion de Vander Waerdt 1993, 31: «Yet it is hard to imagine a more provocative comparison than to impute divinity to the father of the regime which had recently destroyed Athens' political power after decades of war".

24. Cf. Hdt. I, 65.

25. Voir aussi Mém. I, 6, 10.

26. Fritz 1931, 65, note la différence entre Xénophon et Platon, mais il l'interprète comme une maladresse de rédaction; voir aussi Breitenbach 1967, col. 1889. 
venir, qui correspond au récit de l'oracle ${ }^{27}$. La première irruption du tumulte n'est donc pas provoquée par les mêmes propos, à la différence de la deuxième, qui correspond globalement à la réponse de l'oracle. Xénophon insiste davantage sur le contexte religieux de la megalègoria en dramatisant la scène: la défense contre l'accusation d'impiété et l'évocation de l'oracle sont dans sa version les points qui choquent le plus l'assistance.

3. Le «tumulte» est souvent associé à l'assemblée démocratique dans le corpus de Platon comme dans celui de Xénophon. On a pu opposer l'idéal de parrhésia et le thorubos, le deuxième étant une limitation du premier, un «negative vote by the people ", qui décide de ce qu'il veut entendre ou ne pas entendre ${ }^{28}$. Cet arrière-plan politique a son importance. Mais le tumulte a surtout une double cause émotionnelle dans le commentaire narratif de Xénophon.

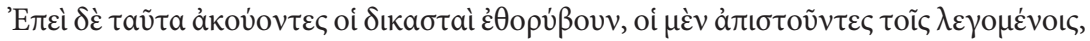

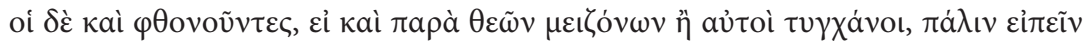

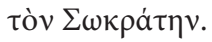

Comme en entendant ces mots les juges faisaient du tumulte, les uns par défiance à l'égard des propos de Socrate, les autres par jalousie surtout de ce qu'il obtînt même de la part des dieux plus qu'eux-mêmes, Socrate reprit la parole (Ap. 14; trad. pers.).

Le tumulte des juges est ainsi expliqué par deux types d'émotions: l'apistia, c'est-àdire la défiance ou l'incrédulité par rapport aux propos prononcés par Socrate, et le phthonos, jalousie à l'idée que Socrate puisse avoir plus qu'eux-mêmes. Le premier sentiment est sous-jacent dans le passage parallèle de Platon; Socrate déclare ainsi dans une précaution de langage qui précède de peu la mention du tumulte: «Peutêtre, il est vrai, quelques-uns vont s'imaginer que je plaisante. Non, croyez-le bien, ce que je vais dire est la pure vérité» (2od). Mais cette réflexion précède l'affirmation qu'il possède une science "propre à l'homme", par opposition à celle des sophistes, qui est «plus qu'humaine». En revanche, dans la version de Xénophon, l'incrédulité porte sur la relation privilégiée entre Socrate et la divinité.

Reste la jalousie. A priori, il semble difficile d'être à la fois incrédule et jaloux, et il est préférable aussi d'un point de vue syntaxique de distinguer deux groupes dont la réaction de rejet est identique, mais pour des raisons différentes. Faut-il aussi accorder une plus grande importance au deuxième, en raison de la présence de l'intensif kai? Les traductions que nous avons consultées ne le font pas.

27. Voir Arnim 1923, 15 et 86.

28. Voir Pontier 2006, ch. 1 et 4; Bers 1985; Van Raalte 2004; Wallace 2004, 225: «In the assembly, thorubos had the practical purpose of regulating debate. [...] Thorubos was a negative vote by the people, constituting the fundamental power of the community to decide what it would listen to". 
Or, dans son compte rendu de l'édition du Banquet de Xénophon dans la CUF $^{29}$, G.B. Kerferd avait noté que le mot kaí n'était pas traduit dans un passage du chapitre I dont la syntaxe est rigoureusement identique à celui de l'Apologie. Xénophon y montre Socrate et ses compagnons se rendant tous chez Callias, les uns après avoir fait de l'exercice et s'être frottés d'huile, les autres allant même jusqu'à prendre un bain, précision que l'on peut interpréter facilement en songeant au bain que, dans la version platonicienne du Banquet, Socrate a pris exceptionnellement avant de se rendre chez Agathon ${ }^{30}$. On peut trouver de nombreux autres passages où la présence de kaí n'est pas anodine, parmi lesquels figure celui de l'Apologie. Elle témoigne souvent d'une gradation ou d'une insistance sur le deuxième volet de la phrase ${ }^{31}$. La nuance peut être une simple addition ou marquer une opposition ${ }^{32}$. Kaí a alors un sens très proche de l'adverbe aṽ, «d'autre part, de leur côté».

Par conséquent, il y a une gradation ou, au moins, une insistance sur le deuxième - et principal? - motif de tumulte d'une partie des juges: la jalousie. Ce thème est discrètement présent dans la version platonicienne, mais de façon plus indirecte, sans que le public présent soit directement concerné, et sans que cela soit associé non plus à la relation privilégiée que Socrate entretient avec la divinité ${ }^{33}$. En revanche, dans un passage récapitulatif de l'Apologie $(\$ 32)$, Xénophon refait allusion aux propos de Socrate en ne retenant comme émotion suscitée chez les juges que la jalousie - ce qui confirme, soit dit en passant, notre analyse de kaí au $₫ 14^{34}$.

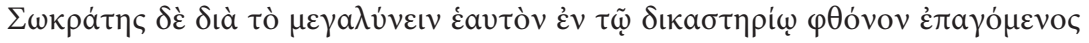

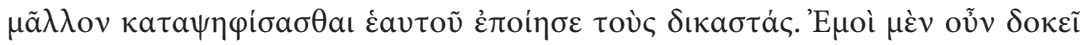

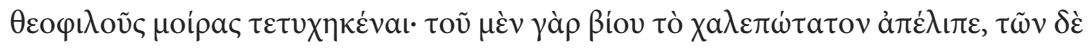

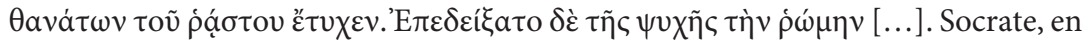
se glorifiant devant le tribunal, s'attira la jalousie des juges et les incita davantage à le condamner. Mais il me semble que son sort fut un bienfait des dieux, puisqu'il a évité la partie la plus pénible de la vie et obtenu la plus facile des morts; et il a aussi déployé la force de son âme [...]. (Ap. 32-33; trad. pers.)

29. Voir Kerferd 1962.

30. Voir Plat., Conv. 174a2. La remarque peut aussi se comprendre par rapport à Xen., Conv. I, 4, et à la réflexion peut-être ironique de Callias.

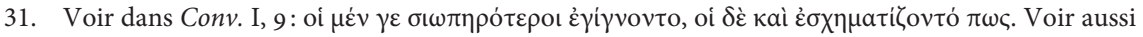
Hell. IV, 4, 11; IV, 5, 14; Mém. I, 3, 1; An. I, 3, 13; V, 3, 10; V, 6, 11, etc.

32. Cf. Denniston 1954, 305: "to supplement the adversative or disjunctive sense with the idea of addition".

33. Voir Ap. $18 \mathrm{~d}$ et $28 \mathrm{a}$, où le sentiment est associé à la "calomnie » et n'a rien à voir avec le « tumulte».

34. Le thème de la jalousie est également présent dans la comparaison que fait Socrate avec la mort de Palamède (26); voir aussi le passage parallèle de Platon (Ap. 41b) et Mém. IV, 2, 33. 
Pourquoi Xénophon insiste-t-il autant sur la jalousie des juges? On peut y voir plusieurs raisons. Il y a peut-être une raison politique. Dans le contexte historique de la fin de la guerre du Péloponnèse, on a pu mettre en évidence l'importance du sentiment de phthonos $^{35}$. Par ailleurs, d'un point de vue rhétorique, il est bien connu que l'éloge de soi a très mauvaise réputation, comme le montre un lieu commun déjà présent chez Pindare:

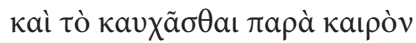

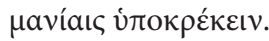

«La vantardise importune accompagne le chant de la folie» (Ol. 9, 38-39; trad. Puech 1949 modifiée).

Et Plutarque, dans son De laude ipsius, donne bien des exemples des dangers de l'éloge de soi, que le mot de Démocrite résume bien : «il vaut mieux être loué par un autre que par soi-même» (DK 68 B 114). Il peut ainsi être mal vu de faire publiquement son propre éloge, à cause de la jalousie ou de la haine qu'il peut susciter. Or, Xénophon connaissait bien ces règles élémentaires de l'éloge, comme en témoigne sa réflexion sur la question dans le reste du corpus. C'est bien pourquoi il a pris la peine d'expliquer pourquoi Socrate décide de s'exprimer ainsi à cause du daimonion et du kairos. Par suite, peut-on éprouver de la jalousie, du moment que l'éloge de soi est juste et fait «à propos»? Et peut-on même être jaloux tout court, si l'on est sage? Il faut faire un détour par les Mémorables pour trouver une définition de la jalousie par Socrate:

Examinant ce qu'était la jalousie, il découvrit qu'elle était une sorte de peine, qui n'est cependant pas celle causée par les revers de fortune de nos amis ou la bonne fortune de nos ennemis. Il affirmait que ceux-là seuls sont jaloux qui s'affligent des succès de leurs amis. [...] Toutefois, cela n'est jamais le fait d'un homme sensé, car

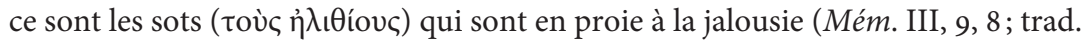
Dorion 2011a modifiée).

Le sage est au contraire celui qui parvient à "éradiquer» (ả Dans la mesure où la jalousie est considérée comme l'apanage des insensés, on peut en déduire que le narrateur de l'Apologie tient ouvertement les juges de l'Apologie pour tels. L'image négative d'un jury propre à se laisser berner, à condamner les innocents et à disculper les coupables, sous-tend de toute façon le récit de Xénophon dès la première conversation de Socrate avec Hermogène ${ }^{37}$. À la logique de

35. Voir Azoulay 2004, 234.

36. Cf. Mém. II, 6, 23, et plus largement 21-24.

37. Cf. Ap. $4 ; 9$ et 23 . 
l'efficacité rhétorique s'oppose donc la défense de l'éthique. À la réprobation et à la condamnation que peut s'attirer un homme qui fait l'éloge de sa propre existence, Xénophon oppose la sottise d'un jury coupable de jalousie. Si la megalègoria de

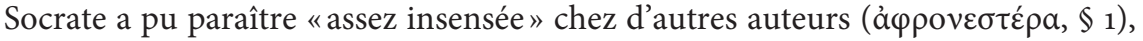
l'intention de Xénophon est de montrer au contraire son côté sensé à l'aide de l'absence d'intelligence du jury. Ainsi, par un renversement du raisonnement, le phthonos devient une donnée essentielle du verdict, dont il remet en cause la probité, alors que la performance de la megalègoria permet à l'opposé de "faire montre ${ }^{38}$ de la force d'âme de Socrate. Le discours de Socrate tient lieu de révélateur de la jalousie du jury. On comprend dès lors la surenchère qu'il semble pratiquer entre les deux manifestations de thorubos: "Allons, écoutez encore autre chose, pour que ceux d'entre vous qui le désirent se défient encore davantage ( $\mu \tilde{a} \lambda \lambda$ ov) de la faveur

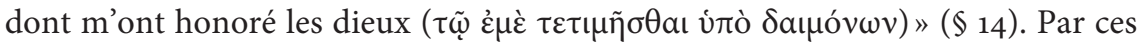
propos, qui sont parfois perçus comme une provocation, Socrate montre seulement sa connaissance de son auditoire en pointant le motif principal de jalousie : la marque d'honneur reçue des dieux ${ }^{39}$.

Par suite, doit-on considérer que le discours d'éloge de soi, en guise d'apologie, est l'équivalent d'un "suicide» par le biais du verdict judiciaire ${ }^{40}$ ? La réalité du texte de l'Apologie est un peu plus ambiguë qu'il n'y paraît ${ }^{41}$; et nous rejoignons la position de Louis-André Dorion, qui refuse de considérer que la megalègoria de Socrate est une stratégie suicidaire destinée à indisposer les juges ${ }^{42}$. L'Apologie, en ne se limitant pas aux propos de Socrate, montre la cohérence de son attitude: il explique aux juges ce qu'il est, conformément à son idéal de conduite, quitte à mourir plutôt que de mendier une prolongation de son existence $(\$ 9)$. En suivant les signes que lui envoie la divinité, Socrate affirme ne rien faire pour provoquer les juges: ce sont eux qui, par faiblesse d'esprit, ont tort d'être envieux et injustes. Si les circonstances - son âge et le signe du dieu - font qu'il considère le «moment»

38. La phrase qui suit l'extrait que nous citons montre que la «force d'âme» de Socrate s'exprime dans les derniers moments de son existence dès le moment où il a pris sa «décision» (है $\gamma \nu \omega)$; la $\mu \varepsilon \gamma \alpha \lambda \eta \gamma$ pía en fait donc partie. Le verbe $\varepsilon \dot{\pi} \varepsilon \delta \varepsilon \dot{\xi} \xi \alpha \tau$ to peut avoir un sous-entendu rhétorique (genre littéraire de l'epideixis), sous-entendu sur lequel Xénophon joue ici. Il y a un passage parallèle dans le livre IV des Mémorables avec la reprise de ce terme (IV, 8, 1).

39. Par cette précision, Xénophon se démarque bien de Platon: dans l'Apologie platonicienne, Socrate explique que la jalousie qu'il s'est attirée porte surtout sur son savoir et sa vertu. Voir Brisson 2000, 226 (et, de façon générale, sur phthonos chez Platon). Enfin, on notera dans le $\$ 14$ l'adverbe $\mu \tilde{\alpha} \lambda \lambda \mathrm{ov}$, comme dans le $\$ 32$, qui marque une gradation dans la réaction de rejet.

40. Voir Vlastos 1994, 397; Azoulay 2004, 272; Waterfield 2012, 270.

41. Voir $\$ 32$, déjà cité p. 68. L'ambiguïté de la phrase tient notamment à l'interprétation de l'adverbe

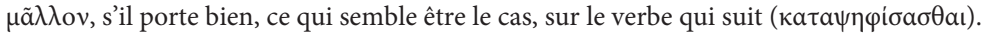

42. Voir Dorion 2013, 306-308. 


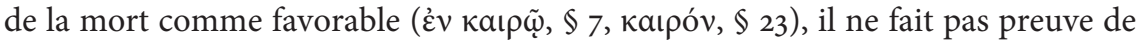
présomption «intempestive», pour reprendre le mot de Pindare. Sa conduite est jugée comme appropriée par Xénophon; elle rejoint les cas positifs de megalègoria occasionnelle que l'on a pu relever dans les portraits élogieux de grands chefs (Cyrus et Agésilas): rappelons que Cyrus est décrit par Phéraulas comme un juge impartial, c'est-à-dire quelqu'un qui «ne juge pas mû par la jalousie» (ov̉ $\varphi \theta o ́ v \omega$ кpível, Cyr. II, 3, 12).

Pour Xénophon, la megalègoria de Socrate a atteint son but. Compte tenu de l'issue du procès du philosophe, un tel raisonnement pouvait à première vue paraître paradoxal; aussi comprend-on que Plutarque n'ait pas retenu l'exemple du philosophe dans ses bons exemples de megalègoria et qu'il ait préféré retenir du corpus de Xénophon les cas d'Agésilas ou de Cyrus ${ }^{43}$. On comprend également les hésitations des traducteurs sur ce terme, même si l'on doit sans doute éviter d'utiliser le terme d' "arrogance $»^{44}$, à moins de se placer uniquement selon le point de vue des juges jaloux.

La megalègoria de Socrate est surtout mise en scène dans le passage où Xénophon note la double réaction des juges au moment du récit de l'oracle. Les raisons du tumulte diffèrent quelque peu, de Platon à Xénophon, tout comme la forme que prend la megalègoria socratique. Le contexte judiciaire et la réflexion sur la recherche des arguments et sur l'élaboration de la ligne de défense montrent bien l'existence d'un débat rhétorique ${ }^{45}$ doublé d'un débat éthique. La défense de Socrate sert de révélateur; si le philosophe a été condamné à mort, ce n'est pas tant à cause de la teneur de sa défense que parce que la justice athénienne se révèle incapable de juger et coupable de phthonos. La prise de position est politique: tout en justifiant l'attitude de Socrate face à ses juges, au nom du signe divin et de la cohérence des paroles et des actes, Xénophon souligne les limites des institutions judiciaires athéniennes et les réactions négatives d'un auditoire mû par sa jalousie et sa médiocrité.

Pierre PonTIER

Université Paris-Sorbonne, Paris IV

43. Cf. Plut., Mor. 545a-b.

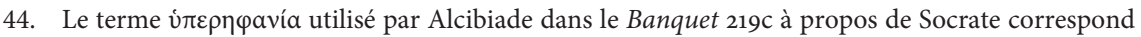
mieux à l'idée d' "arrogance», mais Xénophon n'en fait pas usage. Nous sommes donc en désaccord avec la lecture que fait Hogenmüller 2011, 176, du $\$ 32$ de l’Apologie. Sa démarche est cependant différente, puisqu'il interprète la megalègoria en s'intéressant au Socrate historique, ce qui est hors de notre propos.

45. Le débat rhétorique porte ici sur la forme particulière d'apologie en tant qu'éloge de soi. Ce genre de débat affleure aussi dans certains discours des Helléniques, où Xénophon accorde toute son attention à la personnalité de l'orateur, à l'interaction entre l'orateur et l'assistance, du procès des stratèges des Arginuses (I, 7) jusqu'aux discours du livre VI notamment. Voir Pontier 2013. 


\section{Références bibliographiques}

Arnim H. von (1923), Xenophons Memorabilien und Apologie des Sokrates, Copenhague, A.F. Høst og søn (Kongelige Danske Videnskabernes Selskabs. Historisk-filologiske meddelelser; 8, 1).

Azoulay V. (2004), Xénophon et les grâces du pouvoir. De la charis au charisme, Paris, Publications de la Sorbonne (Histoire ancienne et médiévale; 77).

Bers V. (1985), "Dikastic thorubos ", in Crux. Essays on Greek History Presented to G.E.M. de Sainte Croix on his $75^{\text {th }}$ Birthday, P.A. Cartledge, F.D. Harvey (éd.), Exeter Londres, Imprint Academic - Duckworth, p. 1-15.

Breitenbach H.R. (1967), «Xenophon von Athen », RE, IX A2, col. 1569-2052 (Sonderdruck: Stuttgart, A. Druckenmüller Verlag, 1966).

Brisson L. (1997), Apologie de Socrate, Criton, Paris, Flammarion (GF; 848).

Brisson L. (2000), «La notion de phthonos chez Platon», in Lectures de Platon, Paris, Vrin (Bibliothèque d'histoire de la philosophie), p. 219-234.

Chambry P. (1967), Xénophon, Euvres complètes, 1: Cyropédie, Hipparque, Équitation, Hiéron, Agésilas, Revenus, Paris, Garnier-Flammarion ( $1^{\text {re }}$ édition: Cyropédie, Hipparque, Équitation, Hiéron, Agésilas, Revenus, Paris, Garnier Frères [Classiques Garnier], 1932).

Chambry P. (1967), Xénophon, Euvres complètes, 3: Les Helléniques, l’Apologie de Socrate, les Mémorables, Paris, Garnier-Flammarion ( $1^{\mathrm{re}}$ édition: Helléniques, Apologie de Socrate, Mémorables, Paris, Garnier Frères [Classiques Garnier], 1935).

Danzig G. (2010), Apologizing for Socrates. How Plato and Xenophon Created Our Socrates, Lanham (MD) - New York, Lexington Books.

Demont P. (2014), «Remarques sur la technique du dialogue dans la Cyropédie», in Pontier 2014, p. 195-210.

Denniston J.D. (1954), The Greek Particles. Second Edition, Revised by K.J. Dover, Oxford, Clarendon Press (1 ${ }^{\text {re }}$ éd. : ibid., 1934).

Dorion L.-A. (2000), Xénophon. Mémorables, t. I: Introduction générale. Livre I, M. Bandini (éd.), L.-A. Dorion (trad.), Paris, Les Belles Lettres (CUF; 399).

Dorion L.-A. (2011a), Xénophon. Mémorables, t. II-1: Livres II et III, M. Bandini (éd.), L.-A. Dorion (trad. et notes), Paris, Les Belles Lettres (CUF; 477).

Dorion L.-A. (2011b), Xénophon. Mémorables, t. II-2: Livre IV, M. Bandini (éd.), L.-A. Dorion (trad. et notes), Paris, Les Belles Lettres (CUF; 478).

Dorion L.-A. (2013), L'autre Socrate. Études sur les écrits socratiques de Xénophon, Paris, Les Belles Lettres (L'Âne d'or; 40). 
Fritz K. von (1931), «Zur Frage der Echtheit der xenophontischen Apologie des Sokrates», $R h M, 80$, p. 36-68.

Gigon O. (1946), «Xenophons Apologie des Sokrates, I», MH, 3, p. 210-245.

Gray V.J. (1989), "Xenophon's Defence of Socrates: The Rhetorical Background to the Socratic Problem», CQ, 39, p. 136-140.

Gray V.J. (2014), «Le style simple de Xénophon: du rabaissement de la grandeur», in Pontier 2014, p. 319-337.

Hobden F., Tuplin C. (éd.) (2012), Xenophon: Ethical Principles and Historical Enquiry, Leyde - Boston, Brill (Mnemosyne, Supplements. History and Archaeology of Classical Antiquity; 348).

Hogenmüller B. (2008), Xenophon, Apologie des Sokrates. Ein Kommentar, Sarrebruck, Südwestdeutscher Verlag für Hochschulschriften.

Hogenmüller B. (2011), "Die Verurteilung des Sokrates. Die Bedeutung des $\mu \varepsilon \gamma a \lambda \eta \gamma$ pía», SO, 85, 1, p. 169-183.

Kerferd G.B. (1962), compte rendu de Ollier 1961, Gnomon, 34, p. 21-24.

KLAERr R. (1974), in Plutarque, Euvres morales, t. VII - $2^{\mathrm{e}}$ partie: De l'amour des richesses. De la fausse honte. De l'envie et de la haine. Comment se louer soi-même sans exciter l'envie. Sur les délais de la justice divine, R. Klaerr, Y. Vernière (éd. \& trad.), Paris, Les Belles Lettres (CUF; 231).

Macleod M.D. (2008), Xenophon: Apology and Memorabilia I. With an Introduction, Translation and Commentary, Oxford, Oxbow Books (Aris \& Phillips Classical Texts).

Masqueray P. (1930-1931), Xénophon, Anabase, t. I (Livres I-III); t. II (Livres IV-VII), P. Masqueray (éd. \& trad.), Paris, Les Belles Lettres (CUF; 54; 61).

NARCy M. (2005), «Socrates sentenced by his daimôn», in Socrates' Divine Sign: Religion, Practice, and Value in Socratic Philosophy, P. Destrée, N.D. Smith (éd.), Kelowna (BC), Academic Printing and Publishing (= Apeiron, A Journal of Ancient Philosophy and Science; 38), p. 113-125.

Ollier F. (1961), Xénophon. Banquet, Apologie de Socrate, F. Ollier (éd. et trad.), Paris, Les Belles Lettres (CUF; 153).

Pontier P. (2006), Trouble et ordre chez Platon et Xénophon, Paris, Vrin (Histoire des doctrines de l'Antiquité classique; 34).

Pontier P. (2013), «L'utilisation de l'histoire dans les discours politiques de Xénophon, de Marathon à Platées », DHA. Supplément 8, p. 165-187.

Pontier P. (dir.) (2014), Xénophon et la rhétorique, Paris, PUPS (Hellenica).

Pucci P. (2002), Socrates' Defense, Amsterdam, A.M. Hakkert (Supplementi di Lexis; 10). 
Puech A. (1949), Pindare, t. I: Olympiques, A. Puech (éd. \& trad.), 3 éd. revue et corrigée, Paris, Les Belles Lettres (CUF; 5).

Richards H. (1898), "The Minor Works of Xenophon: X. Apologia Socratis », CR, $12 / 4$, p. $193-195$.

Shero L.R. (1927), «Plato's Apology and Xenophon's Apology», CW, 20, p. 107-111.

Sluiter I., Rosen R.M. (éd.) (2004), Free Speech in Classical Antiquity, Leyde, Brill (Mnemosyne. Supplementa; 254).

Stokes M. (2012), «Three Defences of Socrates: Relative Chronology, Politics and Religion ", in Hobden \& Tuplin 2012, p. 243-268.

Tаlвoт E. (1859), Euvres complètes de Xénophon, Paris, L. Hachette et $C^{\text {ie }}$.

Todd O.J. (1922), in Xenophon, Anabasis, Books IV-VII, and Symposium and Apology, C.L. Brownson, O.J. Todd (trad.), Cambridge (Mass.) - Londres, Harvard University Press - W. Heinemann Ltd (LCL).

VAnder Waerdt P.A. (1993), "Socratic Justice and Self-Sufficiency. The Story of the Delphic Oracle in Xenophon's Apology of Socrates», OSAPh, 11, p. 1-48.

VAnder WAerdt P.A. (1994), The Socratic Movement, Ithaca (NY), Cornell University Press.

Van RaAlte M. (2004), «Socratic Parrhesia and its Afterlife in Plato’s Laws », in Sluiter \& Rosen 2004, p. 279-312.

Vlastos G. (1994), Socrate. Ironie et philosophie morale, Paris, Aubier (trad. fr. par C. Dalimier de Socrates, Ironist and Moral Philosopher, Ithaca, Cornell University Press (Cornell Studies in Classical Philology, The Townsend Lectures) - Cambridge, Cambridge University Press, 1991).

Wallace R.W. (2004), "The Power to Speak - and not to listen - in Ancient Athens», in Sluiter \& Rosen 2004, p. 221-232.

Waterfield R. (2012), "Xenophon on Socrates' Trial and Death », in Hobden \& Tuplin 2012, p. 269-306.

Wilamowitz-Moellendorff U. von (1897), «Die xenophontische Apologie», Hermes, 32, p. 99-106. 